\title{
Colony-Stimulating Factor-1 Receptor in the Polarization of Macrophages: A Target for Turning Bad to Good Ones?
}

\section{Elisabetta Rovida* and Persio Dello Sbarba}

Department of Biomedical Sciences Experimental and Clinical "Mario Serio", Section of Pathology and Experimental Oncology, University of Florence and the Tuscan Cancer Institute, Italy

"Corresponding author: Elisabetta Rovida, Department of Biomedical Sciences Experimental and Clinical "Mario Serio", Section of Pathology, University of Florence, Viale G.B. Morgagni 50, Firenze I-50134, Italy, Tel: 390552751320; Fax: 39055432431; E-mail: elisabetta.rovida@unifi.it

Received date: October 20, 2015; Accepted date: December 21, 2015; Published date: December 28, 2015

Copyright: (C 2015 Rovida E, et al. This is an open-access article distributed under the terms of the Creative Commons Attribution License, which permits unrestricted use, distribution, and reproduction in any medium, provided the original author and source are credited.

\begin{abstract}
Macrophages, which are found in all tissues, are an essential component of the innate immune system, and they play important roles in host defense, inflammation, autoimmune diseases as well as cancer. Functionally, macrophages are classified into two types: classically-activated M1 macrophages and alternatively-activated M2 macrophages. The M1 macrophages typically produce high levels of proinflammatory cytokines and chemokines, whereas M2 macrophages show an efficient phagocytic and scavenging activity. Because the phenotypes of polarized M1 and M2 macrophages can be induced, and reversed to some extent, by various signals, different phases of many diseases are associated with dynamic changes in the balance between M1 and M2 macrophages. The colony-stimulating factor 1 receptor (CSF-1R), a class III receptor tyrosine kinase, sustains the survival, proliferation and differentiation of monocytes and macrophages. Drugging CSF-1R may be the only way to target macrophages within a pathological context. However, CSF-1R-dependent signals may be either positive or detrimental depending on the disease and even on the phase of disease. The role of CSF-1R and its ligands, the colony-stimulating factor-1 and interleukin-34, in macrophages with respect to the pathogenesis of several inflammatory or neoplastic diseases has been reviewed previously. This review will focus specifically on evidences obtained about the role of CSF-1R in macrophage polarization in the context of physiological as well as pathological conditions including inflammation and cancer. The possibility to target CSF-1R, using the several inhibitors already available, for the treatment of inflammatory diseases as well as cancer will be also discussed.
\end{abstract}

Keywords: c-Fms; Macrophage activation; M-CSF, macrophage colony-stimulating factor; M-CSFR, macrophage colony-stimulating factor receptor; Cancer; Inflammation

\begin{abstract}
Abbreviations
AKI: Acute Kidney Injury; BM: Bone Marrow; BMDM: BMDerived Macrophages; ccRCC: clear cell Renal Cell Carcinoma; CNS: Central Nervous System; CSF-1: Colony Stimulating Factor-1; CSF-1R: CSF-1 Receptor; DC: Dendritic Cell; DT: Diphtheria Toxin; ERK: Extracellular-signal-Regulated Kinase; FR $\beta$ : Folate Receptor $\beta$; GFAP: Glial Fibrillary Acidic Protein; GBM: Glioblastoma; GM-CSF: Granulocyte Macrophage Colony-Stimulating Factor; HIV: Human Immunodeficiency Virus; HIVE: HIV-Induced Encephalitis; IFN $\gamma$ : Interferon $\gamma$; IL: Interleukin; I/R: Ischemia/Reperfusion; LCCM: L929 Cell-Conditioned Medium; LPS: Lipopolysaccharide; mAb: Monoclonal Antibody; M-CSF: Macrophage Colony-Stimulating Factor; M-CSFR: M-CSF Receptor; MAPK: Mitogen-Activated Protein Kinase; MMTV/PyMT: Mouse Mammary Tumor Virus/Polyoma Middle T; PBMC: Peripheral Blood Mononuclear Cells; PI3K: Phosphatidyl Inositol 3-Kinase; PTX: Paclitaxel; SAA: Serum Amyloid A; SBS: Sickness Behavior Syndrome; SCF: Stem Cell Factor; SIV: Simian Immunodeficiency Virus; SIVE: SIV-Induced Encephalitis; TGF $\beta$ : Transforming Growth Factor $\beta$; TLR: Toll-Like Receptors; TAM: Tumor-Associated Macrophages; TNFa: Tumor Necrosis Factor
\end{abstract} a

\section{Macrophage Polarization}

Monocytes are released from the bone marrow (BM) into the circulation and migrate into almost every tissue of the body, where they differentiate into mature macrophages. Macrophages include liver Kupffer cells, brain microglia, lung alveolar macrophages, peritoneal macrophages, adipose tissue macrophages and bone osteoclasts [1]. Monocytes/macrophages are critical effectors and regulators of a number of patho-physiological processes within innate and adaptive immunity, systemic metabolism, hematopoiesis, angiogenesis, reproduction and cancer [2-4]. Macrophages are highly plastic cells that change phenotype to acquire different skills depending on the microenvironmental stimuli to which they are exposed.

Microbial stimuli, including toll-like receptor (TLR) ligands, the prototype of which is lipopolysaccharide (LPS), and Th1 cytokines like interferon gamma (IFN $\gamma$ ) induce the "classical" macrophage activation (M1-like macrophage polarization). These cells show increased expression of inflammatory cytokines, chemokines, and reactive nitrogen/oxygen intermediates. M1 cells preferentially promote Th1 effector response, possess anti-microbial ability, protect against various types of bacteria and viruses, and display tumoricidal functions. On the other hand, an "alternative" activation (M2-like macrophage polarization) may be elicited by Interleukin (IL)-4 and IL-13 (M2a), immune complexes (M2b), the anti-inflammatory cytokine IL-10 (M2c), transforming growth factor $\beta$ (TGF $\beta$ ) and/or glucocorticoids. Alternatively activated macrophages typically show increased expression of scavenging receptors and scavenging activity, reduced 
expression of inflammatory cytokines, and metabolize arginine to ornithine preferentially via arginase. Moreover, these cells are mainly devoted to tissue repair-remodeling functions and are important for the clearance of parasite infection. Additionally, M2 cells may also be involved in tumor promotion. M1/M2 polarization thus mirrors Th1/Th2 polarization and mediates Th1/Th2 immune responses. Nevertheless, phenotypic and functional abilities of M1 and M2 macrophages are not rigidly segregated, rather representing a continuum ranging from M1 to M2. Furthermore, mouse and human macrophages are distinct with respect to the expression levels of some markers of polarization [5-8].

\section{The Colony-Stimulating Factor-1 Receptor in Macrophage Development and Differentiation}

The colony-stimulating factor- 1 receptor (CSF-1R, also known as macrophage colony-stimulating factor receptor, M-CSFR) is a homodimeric type III receptor tyrosine kinase that is encoded by the c-fms proto-oncogene. It contains an extracellular ligand-binding domain, a transmembrane domain, and an intracellular domain that contains a split kinase domain. CSF-1R regulates the differentiation of myeloid progenitors into heterogeneous populations of monocytes, macrophages, dendritic cells (DC) and bone-resorbing osteoclasts [9]. Moreover, activated CSF-1R promotes the survival, proliferation, differentiation and chemotaxis of differentiated macrophages. The known CSF-1R ligands are CSF-1 (also known as macrophage colonystimulating factor, M-CSF) and IL-34 [10,11]. Although there are significant differences in their signaling through CSF-1R, it is primarily the different expression of CSF-1 and IL-34 in vivo that controls their different spatiotemporal action through CSF-1R. Moreover, CSF-1 exists in several isoforms, which show different, yet sometimes overlapping, roles: a secreted glycoprotein and a secreted proteoglycan, both of which circulate, and a membrane-spanning, cell-surface glycoprotein. The amount of circulating CSF-1 is humorally-regulated. Nevertheless, CSF-1 serum concentration is around $10 \mathrm{ng} / \mathrm{ml}$ in healthy people [12]. By contrast, IL-34 is not detectable in the circulation of healthy individuals, so that IL-34 action is likely restricted to the microenvironments where it is released [11]. CSF-1R appears to be the only receptor for CSF-1, because all of the CSF-1 deficiency phenotypes are exhibited by CSF-1R-deficient mice [13]. On the other hand, IL-34 acts also through the protein tyrosine phosphatase-z receptor [14].

The biological activities of CSF-1R have been extensively characterized, especially those following its engagement with CSF-1 in myeloid cells. CSF-1 binding leads to CSF-1R auto-phosphorylation on many tyrosine residues. Eight tyrosines are known to be phosphorylated in the intracellular domain of the activated murine CSF-1R proto-oncoprotein/oncoprotein: Tyr-559 and Tyr-544, in the juxtamembrane domain, Tyr-697, Tyr-706, and Tyr-721 in the kinase insert domain and Tyr-807, Tyr-921 and Tyr-974 in the carboxyterminal tail [11]. Phosphorylation of individual CSF-1R tyrosines creates docking sites for several signaling molecules thus leading to the activation of signaling pathways. It is well established that phosphorylation of Tyr-559 determines the activation of Src-Family Kinases and of the mitogen-activated protein kinase (MAPK) extracellular-signal-regulated kinase (ERK) 5 (through Tyr-561 of human CSF-1R that corresponds to Tyr-559 in the murine CSF-1R), while phosphorylation of Tyr-721 activates phosphatidyl inositol 3kinase (PI3K). On the other hand, phosphorylation at different sites including that of Tyr-807 activates the MAPK ERK1 and ERK2, while recruitment of Grb2 by phosphorylated Tyr-921 or Tyr-697 may lead to the activation of other signaling pathways [11].

Referring to the relationship of CSF-1R-elicited signals to macrophage polarization, it is known that the PI3K pathway, which is activated by CSF-1R, regulates M1/M2 activation programs in macrophages [15]. Along this line, it was recently reported that pTyr-721 signaling downregulates proinflammatory genes, including IL-1 $\beta$ (a cytokine typically expressed by M1 polarized macrophages), while upregulating the expression of the M2 genes coding for Arginase (Arg1) and IL-10 [16]. Moreover, miR-21 emerged as a CSF-1-induced pTyr-721- and PI3K-dependent product involved in the regulation of macrophage activation. Indeed, miR-21 inhibition impairs the CSF-1dependent reduction of IL- $1 \beta$ expression. Besides suppressing the proinflammatory phenotype in vitro, miR-21 attenuates the recruitment of Ly6Chigh (i.e., inflammatory) monocytes to the peritoneal cavity in response to LPS in vivo. Taken together, these results indicated that pTyr-721 signaling suppresses the macrophage proinflammatory phenotype [16].

Besides ligand-induced tyrosine phosphorylation, CSF-1R activity may be regulated by ligand-induced down-regulation or downmodulation that occurs by ectodomain shedding. In the latter scenario, CSF-1R protein degradation follows protein kinase C activation by TLR-activating molecules, such as LPS, or by IL including IL-4 and IL-2 [17-19]. Whether CSF-1R down-modulation affects macrophage plasticity and response to M1/M2-polarizing stimuli has not been addressed. However, it should be noted that either M1- (LPS, IL-2) and M2-like inducing agents (IL-4) are able to down-regulate CSF-1R [20]. Another important point that should be taken into consideration, especially when thinking about the possible targeting of CSF-1R, is the fact that CSF-1R signaling may be activated, in a ligand-independent manner, following transactivation by other stimuli including prostaglandin E2, a key mediator of immunity, inflammation and cancer, following engagement to its G-protein-coupled E-prostanoid receptors [21].

The essential role of CSF-1/CSF-1R axis in the development of most tissue macrophages has been well-established using naturally occurring csf1-deficient op/op mice [22,23] and csflr knockout mice [13]. Moreover, during early myeloid differentiation, CSF-1 synergizes with other growth factors, including stem cell factor (SCF) and IL-3, to generate, from hematopoietic stem cells, mononuclear phagocyte progenitor cells $[24,25]$. The proliferation and differentiation of these cells to monocytes and macrophages is then regulated by CSF-1 itself, which also controls the survival, proliferation and function of fully differentiated macrophages [24].

The role of CSF-1R in tissue development and homeostasis has been extensively reviewed [26-29]. Recent reports identified a role for CSF-1R in macrophage activation/polarization under physiological conditions. Microglia, the macrophages resident in the central nervous system (CNS), is absent in csflr-deficient mice [30], while csf1deficient mice show decreased microglial numbers and IL34-deficient mice an even more severe lack [31,32]. Increased levels of CSF-1 together with increased microglial cell number and activation are found in many different CNS pathologies including tumors, degenerative diseases and injuries [33,34]. To test the hypothesis that increased CSF-1 levels impact on both microglial cell numbers and phenotype in vivo, a mice model was developed that overexpresses csf 1 in the glial fibrillary acidic protein (GFAP) compartment (i.e. astrocytes) [35]. Compared with controls, csfl-overexpressing mice harbor significantly greater numbers of microglia cells (i.e. CD11b+ 
cells) in brain and increased microglia proliferation. Pharmacological Inhibition of CSF-1R with PLX3397 (Table 1) promotes microglial apoptosis and reverses the microglial increase observed in csfloverexpressing mice. CSF-1 overexpression does not promote basal activation in vivo but causes defects in response to LPS. Indeed, when several parameters linked to macrophage activation were measured $e x$ vivo in microglia from mice systemically-treated with LPS, csfloverexpressing mice were found to underexpress, when compared to control mice, M1-type molecules such as IL- $1 \beta$ and iNos/NOS2 but also the M2 marker IL-10. These results are at variance with several reports indicating that CSF-1 acts synergistically with LPS to induce the expression of several cytokines in monocytes/macrophages. In order to explain these differences, the authors proposed that microglia behaves differently from other macrophage populations on which most of the studies are based [35].

In keeping with a primary role of CSF-1 in monocyte/macrophage development, it was recently reported that endothelial cells that express CSF-1 are able to support the growth of murine BM hematopoietic cells [45]. Endothelial cells and macrophages are known to interact, thereby contributing to the modulation of vascular function [29]. The long-term co-culture of murine endothelial cells immortalized from different tissues with murine hematopoietic cells (in the presence of cytokines such as SCF, Flt3-L, IL-6, IL-3, thrombopoietin and vascularendothelial growth factor A) allows the development of colonies of differentiated macrophages (F4/80+ and Mac1+ cells). Under the above-described conditions, immortalized endothelial cells express the membrane form of CSF-1, while cells of developed colonies express CSF-1R. The functional contribution of CSF1/CSF1R in the expansion, rather than the formation, of these colonies was established using the CSF-1R inhibitor GW2580. Colony macrophages express high levels of M2 markers including Arg1, CD206/Mrc1 and CD36, while no detectable M1 markers (IL-12, IL-1, and tumor necrosis factor $\alpha$, $\mathrm{TNF} \alpha$ ) were found. However, whether CSF-1R was involved in M2 polarization was not addressed [45].

According to the fact that CSF-1 alone is able to sustain monocyte/ macrophage differentiation, the experimental characterization of macrophage activation and functions has been performed mainly using macrophages obtained through CSF-1-induced differentiation of murine $\mathrm{BM}$ or human peripheral-blood monocytes (PBMC). In this respect it should be taken into consideration that the use of human recombinant CSF-1 (hrCSF-1) or of L929 cell-conditioned medium (LCCM) as a source of CSF-1 [46] may lead to different outcomes. Indeed, a different macrophage activation status may arise depending on the source of CSF-1 used, as described for the response to $A$. fumigatus, where hrCSF-1 exhibited a lower effect than LCCM in the predisposition to M1 phenotype [47]. These differences are likely due to the fact that LCCM contains, other than CSF-1, variable amounts of several growth factors and cytokines, including granulocyte macrophage colony-stimulating factor (GM-CSF) and type I interferons, that can cause additional effects in subsequent activation experiments $[47,48]$.

Peritoneal macrophages (resident or elicited) from mice and GMCSF-cultured macrophages from murine BM are also widely used. However, GM-CSF- and CSF-1-differentiated macrophages have different transcriptomes [49] and GM-CSF and CSF-1 may promote alone an M1 or an M2 phenotype, respectively [50,51]. In spite of this, to refer to GM-CSF and CSF-1 as the cytokines responsible for the prototypical M1 or M2 phenotype in not based on solid evidence [7]. Another important point is that CSF-1- and GM-CSF-differentiated macrophages may react similarly to a further polarization induced by IFN $\gamma$ or IL-4+IL-13 [8,52]. In this respect, it has been recently shown that the most robust M2 activation phenotype is induced by IL-4/ IL-10/TGF $\beta$ following differentiation with CSF-1 [53]. Indeed, flow cytometry showed that the expression of the M2-like CD206 mannose receptor as well as of other M2 markers (including the costimulatory receptor CD273, the gene CiiTA, the class B scavenger receptor SRB1, the mannose receptor CD206, the IL-1 receptor 2 (IL-1R2) and hemeoxygenase (HO)-1) were best induced following differentiation with CSF-1 rather than GM-CSF. On the other hand, CSF-1-differentiated macrophages are capable to undergo a later polarization towards the M1 phenotype [8]. While the costimulatory receptor CD86 was elevated following LPS/IFN $\gamma$-induced M1 activation, with highest expression on GM-CSF-differentiated macrophages, the expression of the costimulatory receptor $\mathrm{CD} 274$ was elevated following M1 activation, but, surprisingly, was highest in CSF-1-differentiated cells. Following IL-4/IL-10/TGF $\beta$-induced M2 activation, the M1-associated markers IL-1 and IL-23 dominated in GM-CSF-differentiated cells, whereas CXCL10, CXCL11, IL-6, IL-12 $\alpha$ and TNFa were higher in CSF-1-differentiated M1 cells. Furthermore, CSF-1-differentiated cells, that are often considered to be M2-polarized, expressed accordingly higher levels of M2-associated IL-10, CCL14 and CD206, which were retained following secondary M1 stimulation. These results indicate that, while $\mathrm{M} 1$ and $\mathrm{M} 2$ activation states can be induced irrespective of a rigid segregation of differentiation signals, it is the balance of these signals (growth factors) which defines the ultimate macrophage activation phenotype [53].

CSF-1R is also able to interfere with IL-32-induced macrophage polarization [54]. IL-32 promotes the differentiation of monocytes into macrophage-like cells and induces the production of various cytokines including TNFa and IL-8, so that it is considered an inducer of M1like macrophages $[55,56]$. While investigating how the co-treatment with CSF-1 and IL-32 affects the M1/M2 ratio, it was found that, in CSF-1-differentiated PBMC cells, a further treatment with IL-32 $\gamma$ or CSF-1 activated MAPK such as p38, JNK and ERK1/2, while the IL-32 $\gamma /$ CSF-1 combination induced a more sustained MAPK activation and increased survival. Moreover, IL-32 $\gamma$ induced the production of IL-6, GROa and IL-8, as well as the expression of the costimulatory molecule CD80, at higher levels than CSF-1. These IL-32 $\gamma$-induced M1-like characteristics were unaffected by the cotreatment with CSF-1. Moreover, IL-32 $\gamma$-treated macrophages showed rather low expression levels of CD86, another M1 marker, compared with CSF-1-treated macrophages. On the other hand, M2like characteristics, including phagocytic activity, and CD14 and CD163 expression were significantly upregulated in IL-32 $\gamma$-treated compared with CSF-1-treated macrophages and CSF-1/IL-32 $\gamma$ cotreatment further upregulated the expression of CD14 and CD163. These results suggest that IL-32 $\gamma$ induces macrophages with both M1 and M2 phenotypes, and that CSF- 1 preferentially accelerates the M2 polarization induced by IL-32 $\gamma$. Thus, CSF-1 seems to have either additive or inhibitory effects on IL-32 $\gamma$ action as far as the M1 and M2 phenotypes are concerned [54].

\section{Role of CSF-1R in the Polarization of Macrophages in Inflammatory and Autoimmune Diseases}

CSF-1R signaling plays important roles in inflammatory/ immunitary diseases, including systemic lupus erythematosus, arthritis, atherosclerosis and obesity [57-59]. Importantly, CSF-1 concentration in serum may be increased in several chronic diseases 
[58] where an autocrine mechanism may also be involved [60]. Macrophage populations elicited by CSF-1R signaling are associated with, and may exacerbate, a broad spectrum of inflammatory and autoimmune diseases. On the other hand, macrophages can also contribute to immunosuppression, disease resolution and tissue repair $[12,28]$.

\section{Pro-inflammatory role of CSF-1R signaling}

Besides inflammation-associated organ dysfunction, patients with infections and autoimmune diseases suffer from sickness behavior syndrome (SBS), which is characterized by fatigue, depression, weight loss and reduced social activities [61]. The interactions of CD40 with its ligand $(\mathrm{CD} 40 \mathrm{~L})$ play a major role in the development of the host response to infectious pathogens and of chronic inflammatory diseases including autoimmune liver diseases. CD40 mediates T cell-dependent $\mathrm{B}$ cell responses, enhances the expression of MHC and costimulatory molecules on DC and macrophages and elicits the production of proinflammatory cytokines including TNF $\alpha$, IL-1 $\beta$, IL-6, typical M1 cytokines, and IL-8 and GM-CSF and CSF-1 [62]. Treatment of mice with the CSF-1R-blocking monoclonal antibody (mAb) M279 protects from CD40-induced SBS (i.e., prevents the decrease of motional activity and body weight) while increasing IL-10 (M2 cytokine) production rather than decreasing the amount of the proinflammatory cytokines such as the M1 cytokines TNFa and IL- 6 . However, CSF-1R blockade did not impair CD-40-induced splenomegaly and hepatitis. Protection from SBS upon CSF-1R inhibition is mediated by the induction of IL-10 expression in inflammatory monocytes, because anti-CSF-1R mAb failed to prevent the development of CD40-induced SBS in IL- $10^{-/}$mice. This study thus indicates that CSF-1R blocking might be a useful tool to prevent SBS, which impairs quality of life in patients, although the overall course of chronic inflammation would not be affected. Moreover, the above results indicate that targeting CSF-1R may lead to a conversion of inflammatory monocytes to a mixed pro-inflammation/pro-resolution cell type [63]. This is in keeping with the fact that the prolonged treatment of mice with the M279 mAb allows the depletion of CSF-1R-expressing resident tissue macrophages in liver, lung broncho-alveolar space, intestinal tract, pancreas and kidney without ablating the development of inflammatory monocytes [40].

Altered macrophage behavior is central to the pathogenesis of chronic obstructive pulmonary disease (COPD) [64]. Serum amyloid A (SAA) is expressed locally in chronic inflammatory conditions such as COPD, where macrophages that do not correspond to the classic M1/M2 paradigm also accumulate. Differentiation of human monocytes with CSF-1 and GM-CSF together with SAA stimulates the M1 cytokines IL-1 and IL-6 concurrently with the M2 markers CD163 and IL-10 more efficiently than in the absence of SAA. Experiments performed in vivo showed an increase of the inflammatory CD $11 \mathrm{c}^{\text {high }}$ / CD11b ${ }^{\text {high }}$ population following SAA challenge. Inhibition of CSF-1R by intranasal pretreatment with the AFS 98 anti-CSF-1R mAb markedly reduces the emergence of this population. These results indicate CSF-1R targeting as a possible tool to prevent this emergence and a novel approach to treat chronic inflammatory conditions associated with persistent SAA expression [65].

Many individuals with human immunodeficiency virus (HIV) infection develop varying degrees of cognitive impairment, collectively termed HIV-associated neurocognitive disorders. Although the pathogenesis of these disorders is not completely understood, activated microglia and macrophages infiltrating CNS are believed to play a prominent role in its development and/or progression [66]. CSF-1 is detectable in cerebrospinal fluid of HIV-infected patients and is believed to support virus production and disease progression [67]. Macrophages accumulate in the perivascular cuffs and within nodular lesions, either lesions being among the histological hallmarks of both HIV-induced and simian immunodeficiency virus (SIV)-induced encephalitis (HIVE and SIVE, respectively) [68]. A recent study identified macrophages as the main source of CSF-1 in SIV-infected rhesus macaques, a relevant animal model for HIV-associated neuropathogenesis [69]. In the same study, IL-34, the alternative ligand of CSF-1R, was found mostly associated with cells scattered in the parenchyma, rather than with cells accumulating around blood vessels and within nodular lesions [69]. In another paper, the same authors explored the potential relationship of CSF-1 and/or IL-34 with M2 activation in SIVE [70]. They found that CD163, a marker of a subclass of M2 macrophages [7], is upregulated in brain of SIV-infected rhesus macaques with SIVE. Moreover, in SIVE brain, accumulating CSF-1positive macrophages are also $\mathrm{CD} 163+$, indicating that they are a source of CSF-1 in SIVE. By contrast, CD163 expression in parenchyma does not colocalize considerably with CSF-1, but does with IL-34. In addition, in the frontal grey matter, the neurons also express CSF-1 and IL-34, and CSF-1 expression is significantly decreased in SIV and even more in SIVE. In vitro experiments showed that treatment with the CSF-1R inhibitor GW2580 decreases the number of CD163+ cells, thus demonstrating that CSF-1R signaling, via either of its ligands, may contribute to sustained M2 activation in brain in SIV infection and SIVE [70]. On the basis of the above results, the authors conclude that, upon CSF-1 ligation, CSF-1R signaling plays an important role in the pathogenesis of HIV-associated neurodegenerative disease, while additional studies are needed to elucidate the potentially divergent roles of CSF-1 and IL-34. IL-34, indeed, may contribute to M2 activation/polarization in the brain, but may also promote neuronal survival.

\section{Anti-inflammatory role of CSF-1R signaling}

Renal tubule epithelia represent the primary site of damage in acute kidney injury (AKI), a process initiated and propagated by macrophage infiltration [71]. Using two murine models of AKI, based on ischemia/reperfusion (I/R)-induced injury or diphtheria toxin (DT)-induced selective proximal tubule injury, Zhang and colleagues examined the role of macrophages/DC (F4/80hi) in the recovery following AKI. In both models, macrophage/DC depletion during the recovery phase increased functional and histological injury and delayed regeneration. After I/R-induced AKI, there was an early increase of inflammatory (M1) monocytes (as detected based on iNOS expression), followed by the accumulation of renal macrophages/DC with a wound-healing (M2) phenotype (increase of Arg1). By contrast, DT-induced AKI only generated an increase of M2 cells. Genetic or pharmacologic (GW2580) inhibition of CSF-1R blocked macrophage/DC proliferation, decreased M2 polarization and inhibited recovery. These findings demonstrated that CSF-1R-mediated expansion and polarization of resident renal macrophages/DC is an important mechanism mediating renal tubule epithelial regeneration after AKI [72]. The same authors, in a very recent study, confirmed and further characterized the protective role of CSF-1R signaling following AKI. In both models of AKI, selective deletion of CSF- 1 expression in the proximal tubule determined decreased M2 polarization, delayed functional and structural recovery and increased tubulointerstitial fibrosis [73]. Therefore, CSF-1, that is abundantly produced in the kidney and specifically at the proximal tubule, stimulates renal 
epithelial cell proliferation directly after AKI [74], but is also an important mediator of macrophage/DC polarization and recovery from AKI [73].

\section{Role of CSF-1R in the Polarization of Macrophages in the Tumor Context}

Tumor-associated macrophages (TAM) are the predominant leukocytes infiltrating solid tumors and can represent up to $50 \%$ of the tumor mass. TAM can exert dual influence of cancer depending on the activation state, with classically activated (M1) and alternatively activated (M2) cells generally exerting antitumor and pro-tumor functions, respectively $[75,76]$. These are extremes in a continuum of polarization states. It is now recognized that TAM, that are recruited by CSF-1 and MCP-1/CCL2, represent the most abundant immunosuppressive cell population in the tumor microenvironment [77]. A number of cell types within the tumor microenvironment as well as tumor cells produce CSF-1 [78] and circulating CSF-1 may increase in several cancer types including that of breast, endometrium and ovary [79-81].

Macrophage infiltration in tumors has been identified as an independent factor predictive of poor prognosis for several cancer entities, colorectal cancer (CRC) being probably the only exception because in this cancer TAM density is significantly associated with enhanced overall survival $[76,82]$. These diverse anti-tumoral or tumor-promoting activities of TAM are likely promoted by distinct TAM subpopulations, linked to different intratumoral microenvironments [76,83]. Indeed, molecularly and functionally distinct TAM subpopulations coexist in tumors, depending on cancer type, stage of tumor progression and location within the tumor tissue [4]. This TAM heterogeneity likely reflects the inherent plasticity of macrophages in response to microenvironmental triggers. The relevant role of CSF-1R in TAM with respect to cancer progression is well acknowledged [12, 27, 84-86]. More importantly, CSF-1/CSF-1R blocking may represent the only truly selective approach to manipulate macrophages in cancer patients. Accordingly, strong reductions of TAM number have been reported in various tumor models upon blocking of CSF-1/CSF-1R signaling using $\mathrm{mAb}$ targeting either CSF-1 or the extracellular domain of CSF-1R, or using chemical inhibitors of CSF-1R tyrosine kinase activity and thereby the downstream signaling [84].

\section{Pro-tumoral activity of CSF-1R signaling in TAM}

The presence of extensive TAM infiltration in clear cell renal cell carcinoma (ccRCC) microenvironment contributes to cancer progression and metastasis by stimulating angiogenesis, tumor growth, and cellular migration and invasion [87]. Immunostaining of human ccRCC specimens showed a correlation between the number of CD163+ M2 macrophages and age, sex, nuclear grade and TNM classification [88]. On the other hand, high levels of CD163+ cell infiltration were significantly associated with poor clinical prognosis in univariate but not multivariate analysis. Culture supernatants from ccRCC cell lines induced M2 polarization of macrophages, as indicated by the increased expression of CD163 and IL-10, two M2 typical markers, following LPS treatment. Stronger results were obtained when direct interaction of ccRCC cells and macrophages was allowed. Primary ccRCC cells consistently express surface CSF-1. Genetic or pharmacological inhibition of CSF-1R (obtained using specific siRNA or GW2580, respectively) or block of CSF-1 binding to CSF-1R suppressed macrophage IL-10 production induced by ccRCC cells.
These results indicate that the signaling initiated by the cross-talk between ccRCC cells and macrophages is mediated by the CSF1/ CSF1R pair. Furthermore, the block of CSF-1R signaling and the consequent reversion of immunosuppressive conditions emerges as a promising approach for anticancer therapy in patients with ccRCC [88].

Although macrophages are endowed with a high level of plasticity, within the tumor microenvironment they mostly facilitate tumor development rather than regression, mainly because they function as immunosuppressive cells. Along this line, skewing of monocyte differentiation from DC to macrophages has been proposed to contribute to immunosuppression [89]. In a study performed to investigate on the possibility that TAM could be redirected toward a DC-like phenotype, it was found that treatment in vitro with GM-CSF, which is able to induce maturation of myeloid cells toward a DC phenotype [90], does not change the expression of M1-type (IL-1 $\beta$, TNFa, and CXCL10) or M2-type (CCL22, CCL17, IL-10, and CD206) markers in $\mathrm{F} 4 / 80^{\text {high }}$ and $\mathrm{CD} 11 \mathrm{~b}^{\text {high }} \mathrm{TAM}$ isolated from abdominal tumors established by inoculating the colon carcinoma cell line MCA38 [91]. Moreover, the combined treatment of TAM with GMCSF and CSF-1R-targeting siRNA did not alter the expression pattern of M1/M2 marker but was able to induce the expression of STAT1, STAT5 and STAT6, which are usually expressed by DC [92]. These findings indicated that GM-CSF administration together with a CSF-1inhibiting treatment could not redirect TAM to a monocyte-derived DC-like phenotype, as determined by M1/M2 marker expression, but was able to modify cell signaling pathways towards a DC-like pattern [91].

Glioblastoma (GBM), the most aggressive form of glioma, has an invariably unfavorable prognosis, as patients respond minimally to current therapies, including surgery, radiation and chemotherapy. TAM are associated with high tumor grade and poor prognosis in gliomas, possibly because, under the influence of glioma cells, microglia creates an immunosuppressive microenvironment which promotes glioma growth [93]. In a mouse model that recapitulates human proneural GBM, in vivo CSF-1R inhibition using the CSF-1R inhibitor BLZ945 significantly increased survival and induced regression of established tumors [94]. However, these effects were not associated with TAM depletion. Rather, GBM-secreted factors, including GM-CSF and IFN $\gamma$, facilitated TAM survival in the context of CSF-1R inhibition and resulted in a repolarization from protumoral M2 to a highly phagocytic M1 phenotype. These results identify TAM as a promising therapeutic target for proneural gliomas and establish the translational potential of CSF-1R inhibition for GBM therapy [94].

Despite the fact that a role for CSF-1/CSF-1R signaling and TAM in breast cancer progression has been well established [27], attempts to obtain the therapeutic depletion of macrophages from tumors using as single agents $\mathrm{mAb}$ or chemical inhibitors targeting CSF-1 or CSF-1R were disappointing $[36,95]$. Nevertheless, the administration to mice of inhibitors of CSF-1R signaling in combination with standard-of-care chemotherapy slows down the growth of primary tumors significantly and reduces pulmonary metastasis [36]. On the basis of these data, phase 1 and 2 clinical studies combining the CSF-1R inhibitor PLX3397 with chemotherapy are currently under way. The mouse mammary tumor virus/polyoma middle T (MMTV/PyMT) transgenic model of luminal B-type mammary carcinoma was used to shed light on the mechanisms by which the CSF-1/CSF-1R pathway and macrophages repress response to cytotoxic therapy in mammary 
carcinoma. It was found that the anti-CSF1 5A1 blocking mAb, alone or in combination with paclitaxel (PTX), depleted mammary tumors from $\mathrm{CD} 11 \mathrm{~b}^{+} \mathrm{F} 4 / 80^{+} \mathrm{MHCII}^{+} \mathrm{Ly}_{6 \mathrm{C}^{-}}$macrophages [96]. Following antiCSF-1 mAb treatment, IL-10 expression was markedly reduced and it turned out to be mostly produced by macrophage cells in MMTV/ PyMT tumors. Moreover, the block of IL-10R by specific antibodies improves response to PTX, in terms of tumor volume in MMTV/ PyMT mice, or of survival in mice following orthotopic transplantation of MMTV/PyMT-derived tumors, or lung metastasis following treatment with IgG1, anti-CSF-1 mAb, anti-IL-10R mAb, PTX, or a combination thereof and similarly to what observed by antiCSF-1mAb/PTX. Improved response to chemotherapy was CD8 ${ }^{+}$Tcelldependent, but IL-10 did not directly suppress CD8 ${ }^{+}$Tcells or alter macrophage polarization. Instead, IL-10R block increased in intratumoral DC the expression of IL-12, which was necessary for improved outcomes. These data indicate that macrophages infiltrating mammary carcinomas are a significant source of IL-10, which in turn suppresses IL-12 production by DC and thereby limits T-cytotoxic responses during chemotherapy. These data identify a CSF-1/IL-10/ IL-12 axis which is suitable for therapeutic targeting [96].

In another study, it was identified a new mechanism that allows macrophages to maintain locally restrained and smoldering inflammation, which is required in angiogenesis and metastasis. Indeed, it was found that IL-34- and CSF-1-differentiated macrophages exhibit most of the phenotypic (i.e., CD14high CD $163^{\text {high }}$ IL-10 $10^{\text {high }}$ IL-12 ${ }^{\text {low }}$ CD86 $6^{\text {low }}$ ) and functional characteristics (i.e., low T-cell costimulatory properties, inhibition of activated effector T-cell functions) of TAM isolated from human ovarian cancer. Moreover, IL-34- and CSF-1-differentiated TAM in human ovarian cancer switch memory Tcells into Th17 cells via membrane IL-1a [97].

A number of papers from Corbís group identified several markers possibly associated to CSF-1-, but not GM-CSF-, differentiated macrophages within the tumor context. Indeed, it was found that CSF-1-, but not GM-CSF-differentiated macrophages express folate receptor beta (FR $\beta$, encoded for by FOLR2) and display folate binding and internalization ability. Therefore, CSF-1 promotes the expression of a functional FR $\beta$ protein, which was therefore proposed to be a further marker of CSF-1-differentiated M2 macrophages. Consistent with a possible role of FR $\beta$ in vivo, FR $\beta$ is functionally expressed in IL-10 mRNA-expressing $\mathrm{CD}^{+}{ }^{+} \mathrm{CD} 8^{+} \mathrm{CD} 163^{+}$melanoma TAM. Other cytokines commonly released by tumors, including IL- 6 alone and IL-10 in combination with CSF-1, upregulate FOLR2 mRNA expression. Moreover, conditioned media from several cancer cell lines are able to induce the expression of FR $\beta$. Incubation with an anti-CSF1 blocking antibody greatly reduced the upregulation of FOLR2 mRNA promoted by ascitic fluid from breast carcinoma or by conditioned medium from tumor-associated fibroblasts or JEG-3 tumor cells. Therefore, CSF- 1 is a major determinant for FR $\beta$ expression on human macrophages and contributes, alone or in combination with other cytokines, to FR $\beta$ cell surface expression on TAM [98]. These results identified a new possible marker for TAM and, more importantly, provided a possible rationale for folate-conjugated drugs in cancer therapy approaches [99]. In another paper, the same authors showed that the heme regulatory molecules CD163 and HO-1 are preferentially expressed by CSF-1-differentiated (M2) macrophages with respect to GM-CSF-differentiated (M1) macrophages [100]. They also showed that M1 macrophages secreted large amounts of functional activin A that promotes the expression of M1 (GM-CSFdifferentiated) markers, impairs the acquisition of M2 (CSF-1differentiated) markers, and down-regulates the production of IL-10.
These results indicate that activin A contributes to macrophage polarization and shapes the inflammatory behavior of macrophages [101]. More recently, it was also found that CSF-1-differentiated macrophages obtained from human PBMC express and produce CCL2, while GM-CSF-differentiated macrophages express CCL2R. $\mathrm{CD} 14^{+}$TAM isolated from ascitic fluid of different types of cancer express CCL2 at an even higher extent that CSF-1-differentiated macrophages [102]. Taken together, the above-described results support further a protumoral activity of CSF-1R signaling.

\section{Anti-tumoral activity of CSF-1R signaling in TAM}

Despite the abundant literature on the many pro-tumoral functions of TAM in several types of cancer, their role in CRC is controversial. Some studies indicated that macrophages in CRC appear to have antitumor activity and are associated with improved disease-free survival. On the contrary, other studies provided evidence that a massive macrophage infiltration is correlated with tumor progression, growth and disease aggressiveness [103]. Nevertheless, we could not find any literature evidence of a role of CSF-1R in macrophage polarization in the context of CRC or other cancer types.

\section{Conclusive Remarks in View of Drugging CSF-1R to Target Macrophage Polarization}

Targeting CSF-1R signal could be undoubtedly beneficial in many conditions, including several types of cancer and chronic inflammations $[12,28,86]$. The block of CSF-1R may represent a truly selective approach and several CSF-1R signaling inhibitors have been developed [86,104]. However, several points are worth to be discussed, especially with respect to the role of CSF-1R signaling in macrophage polarization.

First, CSF-1R-elicited signals are important for several homeostatic mechanisms, so that their prolonged inhibition might be generally detrimental, although necessary for the treatment of specific diseases such as cancers or chronic inflammations. For example, CSF-1 is a main regulator of osteoclast differentiation, as evidenced by the osteopetrotic phenotypes of CSF-1- or CSF-1R-deficient mice [13,22]. Therefore, different ways to inhibit CSF-1R-signalling should be used to achieve different outcomes. In this respect, using antibodies that specifically prevent the binding to CSF-1R of CSF-1 but not IL-34, or vice versa, would block only specific effects. In this respect, it is worth pointing out that anti-CSF-1R mAb that impair CSF-1 but not IL-34 binding to CSF-1R have been identified [44]. These differences have been explained by supposing that IL-34 and CSF-1 bind overlapping yet different domains of CSF-1R. For example, it has been reported that either CSF-1- or IL-34-differentiated macrophages exhibit most of the phenotypic and functional characteristics of TAM isolated from human ovarian cancer [105]. However, IL-34 and CSF-1-differentiated macrophages may have different biological activities [44]. Another approach to avoid extreme toxicities due to systemic and persistent CSF-1R inhibition led to the development of the new H27K15 antiCSF-1R monoclonal antibody, that, differently from other anti-CSF-1R $\mathrm{mAb}$, does not compete with ligand binding and exhibits different effects on signal transduction and cellular trafficking [41]. First, the $\mathrm{H} 27 \mathrm{~K} 15 \mathrm{mAb}$ downregulates osteoclast differentiation and activity, which could block metastasis-induced bone degradation. Second, it inhibits monocyte differentiation into $\mathrm{CD} 163^{+} \mathrm{CD} 64^{+} \mathrm{M} 2$-polarized suppressor macrophages, rather driving their differentiation towards CD14-CD1a ${ }^{+}$DC. Third, this antibody differs from other anti-CSF-1R $\mathrm{mAb}$ by affecting monocyte survival only marginally. Therefore, the 
H27K15 mAb shows interesting properties that may make it suitable for clinical uses in cancer therapy [41].

On the other hand, in some specific context, the inhibiting strategy (antibodies versus chemical inhibitors) could be chosen based on the kind of molecule preferred in relation to the tissue to be targeted. Importantly, small molecule inhibitors that are able to cross the bloodbrain barrier have been developed (Table 1). Referring to small molecule inhibitors that act at the level of CSF-1R kinase activity, they would also prevent ligand-independent CSF-1R activation, which may be induced via the crosstalk with other signaling pathways [21]. However, $\mathrm{mAb}$ that are able to prevent CSF-1R dimerization have also been developed; these $\mathrm{mAb}$ are also able to prevent ligandindependent CSF-1R activation if it involves receptor dimerization [106]. Another important fact that should be taken into account when choosing the inhibiting treatment is that CSF-1 is cleared from serum by receptor-mediated endocytosis [60], so that the use of antibodies which prevent CSF-1 to CSF-1R binding causes massive elevation in circulating CSF-1. A similar elevation may also result from the killing of CSF-1R-expressing cells. On the other hand, the increase in circulating CSF-1 is not supposed to occur if kinase inhibitors are added, unless they are deadly (see above), because receptor-mediated internalization does not require CSF-1R kinase activity.

Another possible approach to target disease-associated macrophages would be to shift a macrophage population towards a more appropriate phenotype. Indeed, skewing of monocyte differentiation from DC to macrophages has been proposed to contribute to tumor-induced immunosuppression [89]. For example, it has been reported that pharmacological [107] or genetic [91] inhibition of CSF-1R, as well as that based on anti-CSF-1R blocking $\mathrm{mAb}$ [41], can change the function or morphology of macrophages and induce DC-like characteristics. Moreover, mounting evidence from a number of different studies in vivo and in vitro has generally indicated that identifying the activated states of macrophages and targeting the macrophage polarization from $\mathrm{M} 1$ to $\mathrm{M} 2$ or vice versa might represent a novel diagnostic or therapeutic strategy for a number of diseases [15]. In these respect, several examples of modulation of macrophage plasticity have been described in this review.

\begin{tabular}{|l|l|l|l|l|l|}
\hline Inhibitor & Type of molecule & Target & Other targets (IC50, $n$ M) & Notes & Reference \\
\hline PLX3397 & Small-molecule inhibitor & CSF-1R & $\begin{array}{l}\text { CSF-1R (20) } \\
\text { KIT (10) }\end{array}$ & Crosses blood-brain barrier & {$[36]$} \\
\hline GW2580 & Small-molecule inhibitor & CSF-1R & & & Crosses blood-brain barrier \\
\hline BLZ945 & Small-molecule inhibitor & CSF-1R & & & {$[37]$} \\
\hline 5A1 & Blocking mAb antibody & CSF-1 & & & {$[38]$} \\
\hline M279 & Blocking mAb antibody & CSF1-R & & Impairs receptor dimerization & {$[40]$} \\
\hline H27K15 & Blocking mAb antibody & CSF-1R & & Blocks either CSF-1 or IL-34 binding [44] & {$[43]$} \\
\hline AFS98 & Blocking mAb antibody & CSF-1R & & Blocks CSF-1 but not IL-34 binding [44] & {$[43]$} \\
\hline 12-2D6 & Blocking mAb antibody & CSF-1R & & & {$[42]$} \\
\hline 2-4A5 & Blocking mAb antibody & CSF-1R & & \\
\hline mAb: Monoclonal Antibody; CSF-1: Colony Stimulating Factor-1; CSF-1R: CSF-1 Receptor & & \\
\hline
\end{tabular}

Table 1: CSF-1R signaling inhibitors cited in the text.

\section{References}

1. Hume DA (2006) The mononuclear phagocyte system. Curr Opin Immunol 18: 49-53.

2. McNelis JC, Olefsky JM (2014) Macrophages, immunity, and metabolic disease. Immunity 41: 36-48.

3. Mantovani A, Biswas SK, Galdiero MR, Sica A, Locati M (2013) Macrophage plasticity and polarization in tissue repair and remodelling. J Pathol 229: 176-185.

4. Ostuni R, Kratochvill F, Murray PJ, Natoli G (2015) Macrophages and cancer: from mechanisms to therapeutic implications. Trends Immunol 36: 229-239.

5. Hume DA (2015) The Many Alternative Faces of Macrophage Activation. Front Immunol 6: 370.

6. Martinez FO, Gordon S (2014) The M1 and M2 paradigm of macrophage activation: time for reassessment. F1000Prime Rep 6: 13 .

7. Murray PJ, Allen JE, Biswas SK, Fisher EA, Gilroy DW, et al. (2014) Macrophage activation and polarization: nomenclature and experimental guidelines. Immunity 41: 14-20.

8. Jaguin M, Houlbert N, Fardel O, Lecureur V (2013) Polarization profiles of human M-CSF-generated macrophages and comparison of M1- markers in classically activated macrophages from GM-CSF and M-CSF origin. Cell Immunol 281: 51-61.

9. Douglass TG, Driggers L, Zhang JG, Hoa N, Delgado C, et al. (2008) Macrophage colony stimulating factor: not just for macrophages anymore! A gateway into complex biologies. Int Immunopharmacol 8: 1354-1376.

10. Lin H, Lee E, Hestir K, Leo C, Huang M, et al. (2008) Discovery of a cytokine and its receptor by functional screening of the extracellular proteome. Science 320: 807-811.

11. Stanley ER, Chitu V (2014) CSF-1 receptor signaling in myeloid cells. Cold Spring Harb Perspect Biol 6.

12. Hume DA, MacDonald KP (2012) Therapeutic applications of macrophage colony-stimulating factor-1 (CSF-1) and antagonists of CSF-1 receptor (CSF-1R) signaling. Blood 119: 1810-1820.

13. Dai XM, Ryan GR, Hapel AJ, Dominguez MG, Russell RG, et al. (2002) Targeted disruption of the mouse colony-stimulating factor 1 receptor gene results in osteopetrosis, mononuclear phagocyte deficiency, increased primitive progenitor cell frequencies, and reproductive defects. Blood 99: 111-120. 
14. Nandi S, Cioce M, Yeung YG, Nieves E, Tesfa L, et al. (2013) Receptortype protein-tyrosine phosphatase $\zeta$ is a functional receptor for interleukin-34. J Biol Chem 288: 21972-21986.

15. Zhou D, Huang C, Lin Z, Zhan S, Kong L, et al. (2014) Macrophage polarization and function with emphasis on the evolving roles of coordinated regulation of cellular signaling pathways. Cell Signal 26: 192-197.

16. Caescu CI, Guo X, Tesfa L, Bhagat TD, Verma A, et al. (2015) Colony stimulating factor-1 receptor signaling networks inhibit mouse macrophage inflammatory responses by induction of microRNA- 21 . Blood 125: e1-13.

17. Dello Sbarba P, Nencioni L, Labardi D, Rovida E, Caciagli B, et al. (1996) Interleukin 2 down-modulates the macrophage colony-stimulating factor receptor in murine macrophages. Cytokine 8: 488-494.

18. Dello Sbarba P, Rovida E, Caciagli B, Nencioni L, Labardi D, et al. (1996) Interleukin-4 rapidly down-modulates the macrophage colonystimulating factor receptor in murine macrophages. J Leukoc Biol 60: 644-650.

19. Dello Sbarba P, Rovida E (2002) Transmodulation of cell surface regulatory molecules via ectodomain shedding. Biol Chem 383: 69-83.

20. Rovida E, Dello Sbarba P (2014) Possible mechanisms and function of nuclear trafficking of the colony-stimulating factor-1 receptor. Cell Mol Life Sci 71: 3627-3631.

21. Digiacomo G, Ziche M, Dello Sbarba P, Donnini S, Rovida E (2015) Prostaglandin E2 transactivates the colony-stimulating factor-1 receptor and synergizes with colony-stimulating factor- 1 in the induction of macrophage migration via the mitogen-activated protein kinase ERK1/2. FASEB J 29: 2545-2554.

22. Yoshida H, Hayashi S, Kunisada T, Ogawa M, Nishikawa S, et al. (1990) The murine mutation osteopetrosis is in the coding region of the macrophage colony stimulating factor gene. Nature 345: 442-444.

23. Wiktor-Jedrzejczak W, Bartocci A, Ferrante AW Jr, Ahmed-Ansari A, Sell KW, et al. (1990) Total absence of colony-stimulating factor 1 in the macrophage-deficient osteopetrotic (op/op) mouse. Proc Natl Acad Sci U S A 87: 4828-4832.

24. Bartelmez SH, Bradley TR, Bertoncello I, Mochizuki DY, Tushinski RJ, et al. (1989) Interleukin 1 plus interleukin 3 plus colony-stimulating factor 1 are essential for clonal proliferation of primitive myeloid bone marrow cells. Exp Hematol 17: 240-245.

25. Kriegler AB, Verschoor SM, Bernardo D, Bertoncello I (1994) The relationship between different high proliferative potential colony-forming cells in mouse bone marrow. Exp Hematol 22: 432-440.

26. Stanley ER, Chen DM, Lin HS (1978) Induction of macrophage production and proliferation by a purified colony stimulating factor Nature 274: 168-170.

27. Sullivan AR, Pixley FJ (2014) CSF-1R signaling in health and disease: a focus on the mammary gland. J Mammary Gland Biol Neoplasia 19: 149-159.

28. Hamilton JA, Achuthan A (2013) Colony stimulating factors and myeloid cell biology in health and disease. Trends Immunol 34: 81-89.

29. Pollard JW1 (2009) Trophic macrophages in development and disease. Nat Rev Immunol 9: 259-270.

30. Ginhoux F, Greter M, Leboeuf M, Nandi S, See P, et al. (2010) Fate mapping analysis reveals that adult microglia derive from primitive macrophages. Science 330: 841-845.

31. Kondo Y, Duncan ID (2009) Selective reduction in microglia density and function in the white matter of colony-stimulating factor-1-deficient mice. J Neurosci Res 87: 2686-2695.

32. Wang Y, Szretter KJ, Vermi W, Gilfillan S, Rossini C, et al. (2012) IL-34 is a tissue-restricted ligand of CSF1R required for the development of Langerhans cells and microglia. Nat Immunol 13: 753-760.

33. Charles NA, Holland EC, Gilbertson R, Glass R, Kettenmann H (2012) The brain tumor microenvironment. Glia 60: 502-514.

34. Dheen ST, Kaur C, Ling EA (2007) Microglial activation and its implications in the brain diseases. Curr Med Chem 14: 1189-1197.
35. De I, Nikodemova M, Steffen MD, Sokn E, Maklakova VI, et al. (2014) CSF1 overexpression has pleiotropic effects on microglia in vivo. Glia 62: 1955-1967.

36. DeNardo DG, Brennan DJ, Rexhepaj E, Ruffell B, Shiao SL, et al. (2011) Leukocyte complexity predicts breast cancer survival and functionally regulates response to chemotherapy. Cancer Discov 1: 54-67.

37. Conway JG, McDonald B, Parham J, Keith B, Rusnak DW, et al. (2005) Inhibition of colony-stimulating-factor-1 signaling in vivo with the orally bioavailable cFMS kinase inhibitor GW2580. Proc Natl Acad Sci U S A 102: 16078-16083.

38. Wang T, Papoutsi M, Wiesmann M, DeCristofaro M, Keselica MC, et al. (2011) Investigation of correlation among safety biomarkers in serum, histopathological examination, and toxicogenomics. Int J Toxicol 30: 300-312.

39. Lokeshwar BL, Lin HS (1988) Development and characterization of monoclonal antibodies to murine macrophage colony-stimulating factor. J Immunol 141: 483-488,

40. MacDonald KP, Palmer JS, Cronau S, Seppanen E, Olver S, et al. (2010) An antibody against the colony-stimulating factor 1 receptor depletes the resident subset of monocytes and tissue- and tumor-associated macrophages but does not inhibit inflammation. Blood 116: 3955-3963.

41. Haegel H, Thioudellet C, Hallet R, Geist M, Menguy T, et al. (2013) A unique anti-CD115 monoclonal antibody which inhibits osteolysis and skews human monocyte differentiation from M2-polarized macrophages toward dendritic cells. MAbs. 5: 736-747.

42. Sudo T, Nishikawa S, Ogawa M, Kataoka H, Ohno N, et al. (1995) Functional hierarchy of c-kit and c-fms in intramarrow production of CFU-M. Oncogene 11: 2469-2476.

43. Sherr CJ, Ashmun RA, Downing JR, Ohtsuka M, Quan SG, et al. (1989) Inhibition of colony-stimulating factor-1 activity by monoclonal antibodies to the human CSF-1 receptor. Blood 73: 1786-1793.

44. Chihara T, Suzu S, Hassan R, Chutiwitoonchai N, Hiyoshi M, et al. (2010) IL-34 and M-CSF share the receptor Fms but are not identical in biological activity and signal activation. Cell Death Differ 17: 1917-1927.

45. He H, Xu J, Warren CM, Duan D, Li X, et al. (2012) Endothelial cells provide an instructive niche for the differentiation and functional polarization of M2-like macrophages. Blood 120:3152-3162.

46. Ladner MB, Martin GA, Noble JA, Wittman VP, Warren MK, et al. (1988) cDNA cloning and expression of murine macrophage colony-stimulating factor from L929 cells. Proc Natl Acad Sci U S A 85: 6706-6710.

47. Gersuk GM, Razai LW, Marr KA (2008) Methods of in vitro macrophage maturation confer variable inflammatory responses in association with altered expression of cell surface dectin-1. J Immunol Methods 329: $157-166$.

48. Warren MK, Vogel SN (1985) Bone marrow-derived macrophages: development and regulation of differentiation markers by colonystimulating factor and interferons. J Immunol 134: 982-989.

49. Fleetwood AJ, Dinh H, Cook AD, Hertzog PJ, Hamilton JA (2009) GMCSF- and M-CSF-dependent macrophage phenotypes display differential dependence on type I interferon signaling. J Leukoc Biol 86: 411-421.

50. Fleetwood AJ, Lawrence T, Hamilton JA, Cook AD (2007) Granulocyte macrophage colony-stimulating factor (CSF) and macrophage CSF dependent macrophage phenotypes display differences in cytokine profiles and transcription factor activities: implications for CSF blockade in inflammation. J Immunol 178: 5245-5252.

51. Lacey DC, Achuthan A, Fleetwood AJ, Dinh H, Roiniotis J, et al. (2012) Defining GM-CSF- and macrophage-CSF-dependent macrophage responses by in vitro models. J Immunol 188: 5752-5765.

52. Kittan NA, Allen RM, Dhaliwal A, Cavassani KA, Schaller M, et al. (2013) Cytokine induced phenotypic and epigenetic signatures are key to establishing specific macrophage phenotypes. PLoS One 8: e78045.

53. Mia S, Warnecke A, Zhang XM, Malmström V, Harris RA (2014) An optimized protocol for human M2 macrophages using M-CSF and IL-4/ IL-10/TGF- $\beta$ yields a dominant immunosuppressive phenotype. Scand J Immunol 79: 305-314. 
54. Osman A, Bhuyan F, Hashimoto M, Nasser H, Maekawa T, et al. (2014) M-CSF inhibits anti-HIV-1 activity of IL-32, but they enhance M2-like phenotypes of macrophages. J Immunol 192: 5083-5089.

55. Netea MG, Lewis EC, Azam T, Joosten LA, Jaekal J, et al. (2008) Interleukin-32 induces the differentiation of monocytes into macrophage-like cells. Proc Natl Acad Sci U S A 105: 3515-3520.

56. Kim SH, Han SY, Azam T, Yoon DY, Dinarello CA (2005) Interleukin-32: a cytokine and inducer of TNFalpha. Immunity 22: 131-142.

57. Hamilton JA (2008) Colony-stimulating factors in inflammation and autoimmunity. Nat Rev Immunol 8: 533-544.

58. Sweet MJ, Hume DA (2003) CSF-1 as a regulator of macrophage activation and immune responses. Arch Immunol Ther Exp (Warsz) 51: 169-177.

59. Chitu V, Stanley ER (2006) Colony-stimulating factor-1 in immunity and inflammation. Curr Opin Immunol 18: 39-48.

60. Bartocci A, Mastrogiannis DS, Migliorati G, Stockert RJ, Wolkoff AW, et al. (1987) Macrophages specifically regulate the concentration of their own growth factor in the circulation. Proc Natl Acad Sci U S A 84 6179-6183.

61. Dantzer R, O'Connor JC, Freund GG, Johnson RW, Kelley KW (2008) From inflammation to sickness and depression: when the immune system subjugates the brain. Nat Rev Neurosci 9: 46-56.

62. Elgueta R, Benson MJ, de Vries VC, Wasiuk A, Guo Y, et al. (2009) Molecular mechanism and function of CD40/CD40L engagement in the immune system. Immunol Rev 229: 152-172.

63. Müller AF, Strauss L, Greter M, Gast H, Recher M, et al. (2015) Neutralization of colony-stimulating factor 1 receptor prevents sickness behavior syndrome by reprogramming inflammatory monocytes to produce IL-10. Brain Behav Immun 48: 78-85.

64. Vlahos R, Bozinovski S (2014) Role of alveolar macrophages in chronic obstructive pulmonary disease. Front Immunol 5: 435.

65. Anthony D, McQualter JL, Bishara M, Lim EX, Yatmaz S, et al. (2014) SAA drives proinflammatory heterotypic macrophage differentiation in the lung via CSF-1R-dependent signaling. FASEB J 28: 3867-3877.

66. Hong S, Banks WA (2015) Role of the immune system in HIV-associated neuroinflammation and neurocognitive implications. Brain Behav Immun 45: 1-12.

67. Haine V, Fischer-Smith T, Rappaport J (2006) Macrophage colonystimulating factor in the pathogenesis of HIV infection: potential target for therapeutic intervention. J Neuroimmune Pharmacol 1: 32-40.

68. Budka H (1991) Neuropathology of human immunodeficiency virus infection. Brain Pathol 1: 163-175.

69. Gerngross L, Fischer T (2015) Evidence for cFMS signaling in HIV production by brain macrophages and microglia. J Neurovirol 21 249-256.

70. Gerngross L, Lehmicke G, Belkadi A, Fischer T (2015) Role for cFMS in maintaining alternative macrophage polarization in SIV infection implications for HIV neuropathogenesis. J Neuroinflammation 12: 58.

71. Chawla LS, Eggers PW, Star RA, Kimmel PL (2014) Acute kidney injury and chronic kidney disease as interconnected syndromes. N Engl J Med 371: 58-66.

72. Zhang MZ, Yao B, Yang S, Jiang L, Wang S, et al. (2012) CSF-1 signaling mediates recovery from acute kidney injury. J Clin Invest 122: 4519-4532.

73. Wang Y, Chang J, Yao B, Niu A, Kelly E, et al. (2015) Proximal tubulederived colony stimulating factor- 1 mediates polarization of renal macrophages and dendritic cells, and recovery in acute kidney injury. Kidney Int 88: 1274-1282.

74. Menke J, Iwata Y, Rabacal WA, Basu R, Yeung YG, et al. (2009) CSF-1 signals directly to renal tubular epithelial cells to mediate repair in mice. J Clin Invest 119: 2330-2342.

75. Biswas SK, Allavena P, Mantovani A (2013) Tumor-associated macrophages: functional diversity, clinical significance, and open questions. Semin Immunopathol 35: 585-600.

76. Lewis CE, Pollard JW (2006) Distinct role of macrophages in different tumor microenvironments. Cancer Res 66: 605-612.
77. Sica A, Schioppa T, Mantovani A, Allavena P (2006) Tumour associated macrophages are a distinct M2 polarised population promoting tumour progression: potential targets of anti-cancer therapy. Eur J Cancer 42: 717-727.

78. Chockalingam S, Ghosh SS (2014) Macrophage colony-stimulating factor and cancer: a review. Tumour Biol 35: 10635-10644.

79. Scholl SM, Lidereau R, de la Rochefordière A, Le-Nir CC, Mosseri V, et al. (1996) Circulating levels of the macrophage colony stimulating factor CSF-1 in primary and metastatic breast cancer patients. A pilot study. Breast Cancer Res Treat 39: 275-283.

80. Kacinski BM, Chambers SK, Stanley ER, Carter D, Tseng P, et al. (1990) The cytokine CSF-1 (M-CSF) expressed by endometrial carcinomas in vivo and in vitro, may also be a circulating tumor marker of neoplastic disease activity in endometrial carcinoma patients. Int J Radiat Oncol Biol Phys 19: 619-626.

81. Chambers SK (2009) Role of CSF-1 in progression of epithelial ovarian cancer. Future Oncol 5: 1429-1440.

82. Zhang QW, Liu L, Gong CY, Shi HS, Zeng YH, et al. (2012) Prognostic significance of tumor-associated macrophages in solid tumor: a metaanalysis of the literature. PLoS One 7: e50946.

83. De Palma M, Lewis CE (2013) Macrophage regulation of tumor responses to anticancer therapies. Cancer Cell 23: 277-286.

84. Laoui D, Van Overmeire E, De Baetselier P, Van Ginderachter JA, Raes G (2014) Functional Relationship between Tumor-Associated Macrophages and Macrophage Colony-Stimulating Factor as Contributors to Cancer Progression. Front Immunol 5: 489.

85. Ries CH, Hoves S, Cannarile MA, Rüttinger D (2015) CSF-1/CSF-1R targeting agents in clinical development for cancer therapy. Curr Opin Pharmacol 23: 45-51.

86. Jinushi M, Komohara Y (2015) Tumor-associated macrophages as an emerging target against tumors: Creating a new path from bench to bedside. Biochim Biophys Acta 1855: 123-130.

87. Santoni M, Massari F, Amantini C, Nabissi M, Maines F, et al. (2013) Emerging role of tumor-associated macrophages as therapeutic targets in patients with metastatic renal cell carcinoma. Cancer Immunol Immunother 62: 1757-1768.

88. Komohara Y, Hasita H, Ohnishi K, Fujiwara Y, Suzu S, et al. (2011) Macrophage infiltration and its prognostic relevance in clear cell renal cell carcinoma. Cancer Sci 102: 1424-1431.

89. Menetrier-Caux C, Montmain G, Dieu MC, Bain C, Favrot MC, et al. (1998) Inhibition of the differentiation of dendritic cells from CD34 $(+)$ progenitors by tumor cells: role of interleukin- 6 and macrophage colonystimulating factor. Blood 92: 4778-4791.

90. Conti L, Gessani S (2008) GM-CSF in the generation of dendritic cells from human blood monocyte precursors: recent advances. Immunobiology 213: 859-870.

91. Kitoh Y, Saio M, Gotoh N, Umemura N, Nonaka K, et al. (2011) Combined GM-CSF treatment and M-CSF inhibition of tumorassociated macrophages induces dendritic cell-like signaling in vitro. Int J Oncol 38: 1409-1419.

92. Welte T, Koch F, Schuler G, Lechner J, Doppler W, et al. (1997) Granulocyte-macrophage colony-stimulating factor induces a unique set of STAT factors in murine dendritic cells. Eur J Immunol 27: 2737-2740.

93. Domingues P, González-Tablas M, Otero Á, Pascual D, Miranda D, et al. (2015) Tumor infiltrating immune cells in gliomas and meningiomas. Brain Behav Immun.

94. Pyonteck SM, Akkari L, Schuhmacher AJ, Bowman RL, Sevenich L, et al. (2013) CSF-1R inhibition alters macrophage polarization and blocks glioma progression. Nat Med 19: 1264-1272.

95. Strachan DC, Ruffell B, Oei Y, Bissell MJ, Coussens LM, et al. (2013) CSF1R inhibition delays cervical and mammary tumor growth in murine models by attenuating the turnover of tumor-associated macrophages and enhancing infiltration by CD8(+) T cells. Oncoimmunology 2: e26968.

96. Ruffell B, Chang-Strachan D, Chan V, Rosenbusch A, Ho CM, et al. (2014) Macrophage IL-10 blocks CD8+ T cell-dependent responses to 
Citation: Rovida E, Sbarba PD (2015) Colony-Stimulating Factor-1 Receptor in the Polarization of Macrophages: A Target for Turning Bad to Good Ones?. J Clin Cell Immunol 6: 379. doi:10.4172/2155-9899.1000379

Page 10 of 10

chemotherapy by suppressing IL-12 expression in intratumoral dendritic cells. Cancer Cell 26: 623-637.

97. Foucher ED, Blanchard S, Preisser L, Descamps P, Ifrah N, et al. (2015) IL-34- and M-CSF-induced macrophages switch memory $\mathrm{T}$ cells into Th17 cells via membrane IL-1a. Eur J Immunol 45: 1092-1102.

98. Puig-Kröger A, Sierra-Filardi E, Domínguez-Soto A, Samaniego R, Corcuera MT, et al. (2009) Folate receptor beta is expressed by tumorassociated macrophages and constitutes a marker for M2 antiinflammatory/regulatory macrophages. Cancer Res 69: 9395-9403.

99. Zhao X, Li H, Lee RJ (2008) Targeted drug delivery via folate receptors. Expert Opin Drug Deliv 5: 309-319.

100. Sierra-Filardi E, Vega MA, Sánchez-Mateos P, Corbí AL, Puig-Kröger A (2010) Heme Oxygenase-1 expression in M-CSF-polarized M2 macrophages contributes to LPS-induced IL-10 release. Immunobiology 215: 788-795.

101. Sierra-Filardi E, Puig-Kröger A, Blanco FJ, Nieto C, et al. (2011) Activin A skews macrophage polarization by promoting a proinflammatory phenotype and inhibiting the acquisition of anti-inflammatory macrophage markers. Blood 117: 5092-5101.

102. Sierra-Filardi E, Nieto C, Domínguez-Soto A, Barroso R, Sánchez-Mateos P, et al. (2014) CCL2 shapes macrophage polarization by GM-CSF and
M-CSF: identification of CCL2/CCR2-dependent gene expression profile. J Immunol 192: 3858-3867.

103. Erreni M, Mantovani A, Allavena P (2011) Tumor-associated Macrophages (TAM) and Inflammation in Colorectal Cancer. Cancer Microenviron 4: 141-154.

104. El-Gamal MI, Anbar HS, Yoo KH, Oh CH (2013) FMS Kinase Inhibitors: Current Status and Future Prospects. Med Res Rev 33: 599-636.

105. Foucher ED, Blanchard S, Preisser L, Garo E, Ifrah N, et al. (2013) IL-34 induces the differentiation of human monocytes into immunosuppressive macrophages. antagonistic effects of GM-CSF and IFN $\gamma$. PLoS One 8: e56045.

106. Ries CH, Cannarile MA, Hoves S, Benz J, Wartha K, et al. (2014) Targeting tumor-associated macrophages with anti-CSF-1R antibody reveals a strategy for cancer therapy. Cancer Cell 25: 846-859.

107. Irvine KM, Burns CJ, Wilks AF, Su S, Hume DA, et al. (2006) A CSF-1 receptor kinase inhibitor targets effector functions and inhibits proinflammatory cytokine production from murine macrophage populations. FASEB J 20: 1921-1923. 